\title{
Electron-Positron Pair Production By Photons In Nonuniform Strong Fields
}

\author{
H. Nitta, ${ }^{1}$ M. Kh. Khokonov, ${ }^{2}$ Y. Nagata, ${ }^{1}$ and S. Onuki ${ }^{1}$ \\ ${ }^{1}$ Department of Physics, Tokyo Gakugei University, Koganei, Tokyo 184-8501, Japan \\ ${ }^{2}$ Department of Physics, Kabardino-Balkarian State University, 360004 Nalchik, Russian Federation
}

(Received 21 March 2004; published 29 October 2004)

\begin{abstract}
A probability of electron-positron pair production by photons in strong nonuniform fields is derived by applying a model trajectory method in the frame of a semiclassical approach. In addition to the well known invariant field parameter $\chi$, a new invariant parameter $\nu$ is introduced to characterize the nonuniformity of the field. For $\nu \gg 1$, the obtained expression is reduced to the uniform-field approximation while it approaches the Bethe-Heitler formula for $\nu \ll 1$. The pair production is predicted for relatively weak external fields where the uniform-field approximation gives no effect. The theory agrees well with the experimental results of crystal-assisted pair production.
\end{abstract}

DOI: 10.1103/PhysRevLett.93.180407

PACS numbers: $12.20 . \mathrm{Ds}, 41.75 . \mathrm{Ht}$

In strong electromagnetic fields, the process of electron-positron pair production by energetic photons is rather different from the well known Bethe-Heitler type [1,2]. Indeed, spontaneous decay of photons into pairs becomes significant when the field tensor $F^{\mu \nu}$ and photon 4-momentum $k^{\mu}$ satisfy $\chi \gtrsim 1$, where $\chi$ is the well known invariant field parameter [1], $\chi=\left(\lambda_{0} / E_{c r}\right) \times$ $\left[\left(F_{\mu \nu} k^{\nu}\right)^{2}\right]^{1 / 2}, E_{c r}=\left(m_{0}^{2} c^{3}\right) /(e \hbar)=1.3 \times 10^{16} \mathrm{~V} / \mathrm{cm}(=$ $\left.1.4 \times 10^{13} \mathrm{G}\right)$ is the critical electric field, $\lambda_{0}$ the Compton wavelength, and $m_{0}$ the electron mass at rest. For example, observations indicate that neutron stars have magnetic fields higher than $10^{12} \mathrm{G}$ where quantumelectrodynamic (QED) processes become rather exotic $[3,4]$. Also, recent development of intense $\mathrm{x}$-ray free electron lasers could provide field strength $E \gtrsim$ $10^{11} \mathrm{~V} / \mathrm{cm}$ in the near future, which opens up the possibility to test other types of pair-production mechanisms by using crossed laser beams [5] or relativistic ions [6].

Under the condition $\chi \gtrsim 1$, full calculations of strongfield QED processes such as radiation and pair production by photons become very complex even in the simplest case of the uniform-field approximation (UFA) [7]. To overcome such difficulty, Bă̌er and Katkov proposed a semiclassical theory of radiation in which the classical trajectory of a particle plays an important role $[8,9]$. The pair-production process in UFA was also derived from the semiclassical radiation formula by using "crossing symmetry" $[1,9]$.

For experimental study of QED in strong external electric fields, oriented crystals have appeared to be very good natural tools as the averaged electric field of an atomic string provides $E \lesssim 10^{12} \mathrm{~V} / \mathrm{cm} \quad[10,11]$. Therefore, $\chi \gtrsim 1$ is satisfied for energetic photons with energies $\hbar \omega \geqslant 10 \mathrm{GeV}$. The UFA was applied to the "crystal-assisted" pair-production process and explained the experimental result very well at $\theta_{\gamma}=0$, where $\theta_{\gamma}$ is the incident angle of the $\gamma$ photon to the crystal axis [10]. However, for $\theta_{\gamma}>\theta_{L}$, UFA is inapplicable because the field nonuniformity becomes significant, where $\theta_{L}$ repre- sents the Lindhard critical angle of channeling [12]. This inapplicability of UFA has caused serious difficulty to analyze the strong-field QED phenomena because the only possible way to obtain quantitative values has been, so far, to perform very complex numerical computations. For example, the authors of Ref. [13] calculated the Baĭer-Katkov formula and demonstrated a good agreement of their numerical results with the experimental ones [14]. However, their computation is not reproducible because unexplained "numerical experiments" were needed in their computation [13]. Similar numerical evaluations were made in Ref. [15] and obtained a good agreement with the experimental results [16], but again the method of numerical calculation was not described.

The purpose of this Letter is to give a simple as well as precise expression of the pair production in strong fields taking into account the nonuniform effect of the fields. The main idea is to use a trial trajectory method developed recently for radiation processes [17]. The method permits us to express the corresponding cross section directly in terms of the interaction potential rather than through the trajectories.

Let us start with the semiclassical radiation formula by Baĭer and Katkov [8,9], which can be rewritten in the following form for the number of photons emitted per unit time

$$
\frac{d N}{d \eta}=\frac{\alpha c}{\pi \lambda_{0} \gamma}\left(J_{1}+J_{2}\right),
$$

where $\eta=\hbar \omega / E, \hbar \omega$ is the energy of the emitted photon, $E$ the initial energy of a radiating particle, $\gamma=E /\left(m_{0} c^{2}\right)$, and $\alpha$ the fine-structure constant. The factors $J_{1}$ and $J_{2}$ are defined by

$$
\begin{aligned}
& J_{1}=\int_{0}^{\infty}\left[1+\frac{\gamma^{2}}{2}\left(\delta \boldsymbol{\beta}_{\perp}\right)^{2}\right] \sin \Delta \frac{d \tau}{\tau}-\frac{\pi}{2}, \\
& J_{2}=\frac{\left(\gamma-\gamma^{\prime}\right)^{2}}{2 \gamma \gamma^{\prime}} \int_{0}^{\infty} \frac{\gamma^{2}}{2}\left(\delta \boldsymbol{\beta}_{\perp}\right)^{2} \sin \Delta \frac{d \tau}{\tau},
\end{aligned}
$$

where $\delta \boldsymbol{\beta}_{\perp}=\boldsymbol{\beta}_{\perp}\left(t_{+}\right)-\boldsymbol{\beta}_{\perp}\left(t_{-}\right), \boldsymbol{\beta}_{\perp}(t)$ is the transverse 
velocity at the time $t, t_{ \pm}=t_{0} \pm \tau / 2, E^{\prime}=E-\hbar \omega$, and $\gamma^{\prime}=E^{\prime} /\left(m_{0} c^{2}\right)$. The phase factor $\Delta(\tau)$ determined by the trajectory of a radiating particle is given by

$$
\Delta(\tau)=\frac{\omega^{*} \tau}{2 \gamma^{2}}-\frac{\omega^{*}}{2 c^{2} \tau}(\delta \rho)^{2}+\frac{\omega^{*}}{2} \int_{t_{-}}^{t_{+}} \boldsymbol{\beta}_{\perp}^{2}\left(\tau^{\prime}\right) d \tau^{\prime},
$$

where $\omega^{*}=\left(\gamma / \gamma^{\prime}\right) \omega$ and $\delta \boldsymbol{\rho}=\boldsymbol{\rho}\left(t_{+}\right)-\boldsymbol{\rho}\left(t_{-}\right), \boldsymbol{\rho}(t)$ being the transverse coordinate.

The pair-production probability is obtained by applying crossing symmetry to Eq. (1) $[1,18]$. By changing the variables as $E \rightarrow-E_{+}, E^{\prime} \rightarrow E_{-}$, and $\omega \rightarrow-\omega$, and multiplying the ratio of the density of final states, $E_{+}^{2} d E_{+} /\left(\hbar^{3} \omega^{2} d \omega\right)$, we obtain the pair-production probability

$$
\frac{d N_{+}}{d \eta_{+}}=\left(\frac{\alpha c}{\pi \lambda_{0}}\right)\left(\frac{m_{0} c^{2}}{\hbar \omega}\right) J
$$

where $d N_{+} / d \eta_{+}$represents the number of produced positrons, $J=J_{1}+J_{2}, \eta_{+}=E_{+} / \hbar \omega$, and $E_{+}\left(E_{-}\right)$is the energy of the positrons (electrons) [19].

Using the quantum synchrotron formula for the calculation of $J$, one obtains the UFA result $[10,20]$

$$
\begin{aligned}
J_{\mathrm{UFA}}= & \frac{1}{\sqrt{3}}\left[\left(\frac{1-\eta_{+}}{\eta_{+}}+\frac{\eta_{+}}{1-\eta_{+}}\right) \mathrm{K}_{2 / 3}\left(\xi^{*}\right)\right. \\
& \left.+\int_{\xi^{*}}^{\infty} \mathrm{K}_{1 / 3}(\lambda) d \lambda\right]
\end{aligned}
$$

where $\quad \xi^{*}=2 /\left[3 \eta_{+}\left(1-\eta_{+}\right) \chi\right], \quad \chi=\left(\lambda_{0} / E_{c r}\right) \times$ $\left[\left(F_{\mu \nu} k^{\nu}\right)^{2}\right]^{1 / 2}=\left(\hbar \omega / m_{0} c^{2}\right)\left(E_{0} / E_{c r}\right)$, and $E_{0}$ is the field strength.

As is well known, the UFA expression of Eq. (6) is derived by assuming that the trajectory of a positron has a constant curvature. Of course, usually a field is not uniform and the trajectory of a positron depends on the local value of the field. A typical situation is that the potential of a field has a maximum at a certain point of closest approach to the field center and then it gradually decreases as a function of the distance from the center. Recently, the present authors introduced a trial trajectory called the "th trajectory" [17] to take into account the effect of such field variation. We have assumed that the trial trajectory is given by the following transverse velocity

$$
\boldsymbol{\beta}_{\perp}(t)=\mathbf{b}_{0}+\mathbf{b} \tanh (t / T),
$$

where $\mathbf{b}_{0}=\left(\boldsymbol{\beta}_{\perp 1}+\boldsymbol{\beta}_{\perp 2}\right) / 2, \mathbf{b}=\left(\boldsymbol{\beta}_{\perp 2}-\boldsymbol{\beta}_{\perp 1}\right) / 2, \boldsymbol{\beta}_{\perp 1}=$ $\boldsymbol{\beta}_{\perp}(t \rightarrow-\infty), \boldsymbol{\beta}_{\perp 2}=\boldsymbol{\beta}_{\perp}(t \rightarrow+\infty)$, and $T$ is the interaction time. The th trajectory asymptotically approaches to the straight paths at $t \rightarrow \pm \infty$ while, at $|t| \lesssim T$, the trajectory is substantially curved. Since Eq. (7) is integrated easily, we obtain an analytic expression for $J$. Combining crossing symmetry with results for nonsynchrotron radiation [17], we obtain,

$$
\begin{aligned}
J_{\mathrm{th}}= & \int_{0}^{\infty} \frac{d \tau}{\tau}\left[\nu^{2}\left(\frac{1-\eta_{+}}{\eta_{+}}+\frac{\eta_{+}}{1-\eta_{+}}\right) \tanh ^{2} \tau-1\right] \\
& \times \sin [\tilde{\xi}(\tau-\mu \tanh \tau)]+\frac{\pi}{2},
\end{aligned}
$$

where $\quad \nu=\gamma_{+} b, \quad b=|\mathbf{b}|, \quad \tilde{\xi}=\nu\left(1+\nu^{2}\right) /\left[\eta_{+}(1-\right.$ $\left.\left.\eta_{+}\right) \chi\right]$, and $\mu=\nu^{2} /\left(1+\nu^{2}\right) . E_{0}$ in $\chi$ is the field strength at the pair-production point. The arguments similar to those given in Ref. [17] permit us to consider Eq. (8) as a good approximation to the precise expressions (1)-(4). It should be noted that, in addition to the invariant field parameter, Eq. (8) has one more Lorentz invariant parameter $\nu$ which represents the field nonuniformity. $\nu$ can be expressed in terms of the local acceleration of the created positron/electron and its higher derivatives[17].

In spite of its simplicity, Eq. (8) is still not very convenient for concrete numerical calculations due to the very rapid oscillations in the integrand. This problem can be avoided by the saddle point method [21]. The precise path of steepest descent for the phase in Eq. (8) has a complicated form, but the correct value can be obtained by integrating Eq. (8) along the approximate path of steepest descent, $y(x)=y_{0}+\lambda x^{2}$, where $\lambda=$ $(2 \nu)^{-1}+\nu / 6, \tan y_{0}=1 / \nu, x=\operatorname{Re} z, y=\operatorname{Im} z$, and $z$ is the time variable $\tau$ in Eq. (8). We obtain,

$$
\begin{aligned}
J_{\text {th }}= & \operatorname{Im} \int_{0}^{\infty}\left[\nu^{2}\left(\frac{1-\eta_{+}}{\eta_{+}}+\frac{\eta_{+}}{1-\eta_{+}}\right) \tanh ^{2} z-1\right] \\
& \times \exp [\tilde{\xi}(z-\mu \tanh z)]\left(1+i \frac{d y}{d x}\right) \frac{d x}{z},
\end{aligned}
$$

where $d y / d x=(1 / \nu+\nu / 3) x$. According to our numerical computations, integrations based on Eq. (9) appear more than a thousand times faster than direct numerical integrations of Eq. (8). As $\nu$ becomes larger, ordinary numerical integration of Eq. (8) becomes more and more difficult and it takes a longer time to obtain a converged result. In contrast, Eq. (9) always converges very fast, being independent of $\nu$.

In Figs. 1-3, we show the $\nu$ and $\chi$ dependence of the pair-production rate $J_{\text {th }}$ calculated with the use of Eq. (9). When $\nu$ becomes larger, $J_{\text {th }}$ approaches to the UFA. On the other hand, when $\nu \ll 1$ is satisfied, $J_{\text {th }}$ gives the BetheHeitler type spectra. The reduction to UFA formula may be easily seen as follows. For $\nu \gg 1$, the factor $\sin [\tilde{\xi}(\tau-$ $\mu \tanh \tau)]$ in Eq. (8) oscillates very rapidly and only the very narrow region $\tau \ll 1$ contributes to the integral. Then, we may approximate as $\tanh \tau \approx \tau-\tau^{3} / 3$ and obtain,

$$
\begin{gathered}
\int_{0}^{\infty} \frac{d \tau}{\tau}\left(A+B \nu^{2} \tanh ^{2} \tau\right) \sin [\tilde{\xi}(\tau-\mu \tanh \tau)] \\
\approx \int_{0}^{\infty} \frac{d x}{x}\left(A+B x^{2}\right) \sin \left[\frac{2}{3} \xi\left(x+\frac{1}{3} x^{3}\right)\right],
\end{gathered}
$$

where $x=\nu \tau$, and $A$ and $B$ are arbitrary constants. Using Eq. (10) it is straightforward to show that Eq. (8) reduces to Eq. (6). On the other hand, it is generally shown that for $\nu=\beta_{\perp} \gamma \ll 1$ (the dipole limit) the Baìer-Katkov for- 


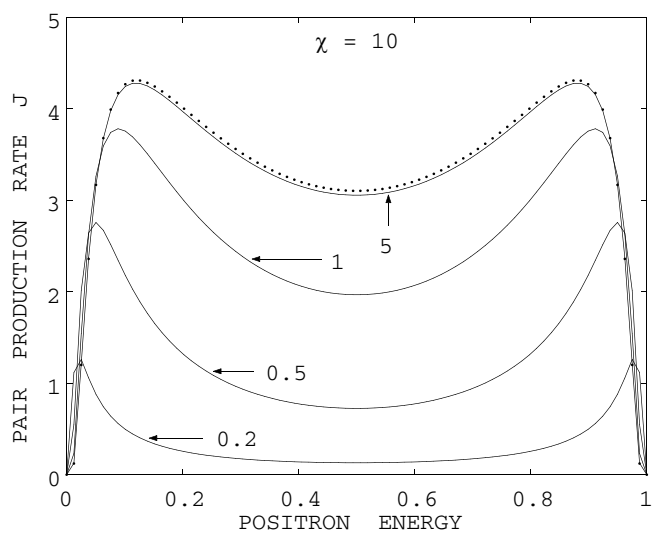

FIG. 1. The $\nu$ dependence of pair-production rate calculated by using Eq. (8) as a function of the energy of produced positrons, $E_{+} / \hbar \omega(\chi=10)$. The dotted line represents the UFA.

mula reproduces the Bethe-Heitler result no matter how the detailed shape of the scattering potential is $[9,20] . J_{\text {th }}$ naturally follows this general tendency for $\nu \ll 1$. It follows from Fig. 3, that UFA does not give pair production for $\chi<0.1$, which corresponds to the region of validity of classical electrodynamics for radiation. We predict, therefore, the pair production even for small $\chi$ where UFA gives negligible probability. It is worthwhile to mention that we may separate the spin contribution by using the same method used in Ref. [22]. Other of our numerical calculations have shown that influence of spin on pair production increases with an increase of $\chi$ whereas its influence decreases with an increase of $\nu$. The influence of spin on the radiation process has been studied recently in [11].

As an application of the pair-production theory developed here, we have calculated the crystal-assisted pairproduction probability as a function of the photon incident angle. In Fig. 4, we compare our results with the experimental ones [14]. Considering both the simplicity of our expression and the fact that there is no fitting parameter, the overall agreements are satisfactory. In the numerical computations, we have employed the one-

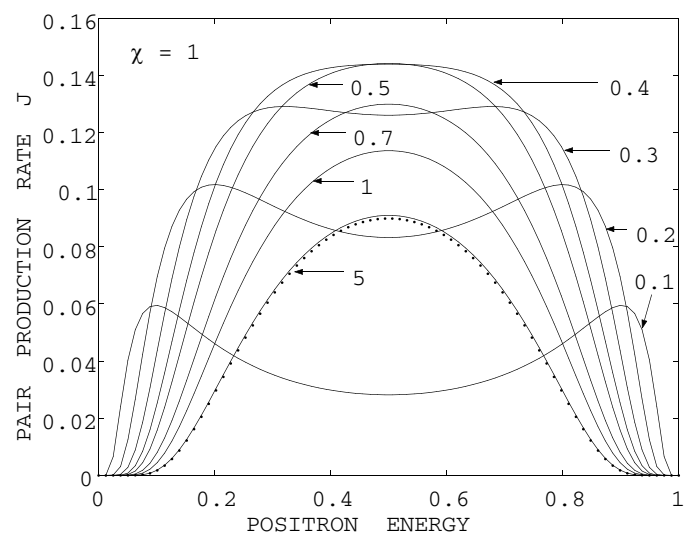

FIG. 2. Same as Fig. 1 , but $\chi=1$.

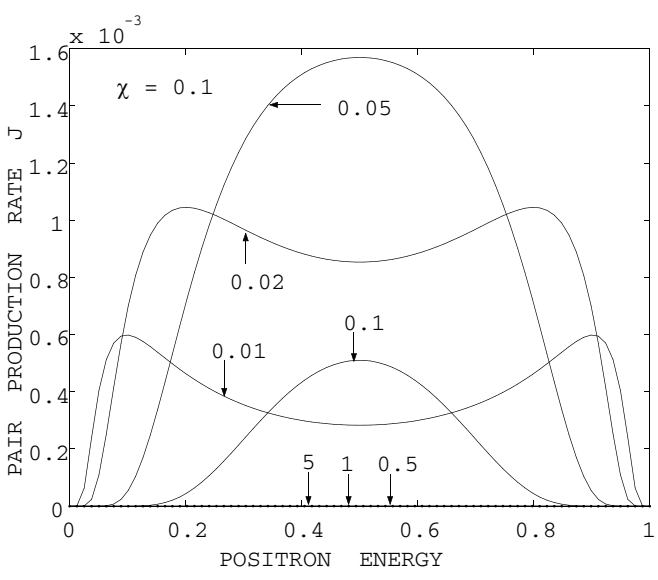

FIG. 3. Same as Fig. 1, but $\chi=0.1$.

string approximation. The area of the string is taken equal to that of a transverse Wigner-Seiz cell of $\mathrm{Ge}\langle 110\rangle$. The photon distribution has been assumed to be homogeneous. The thermally averaged Molière potential with the Debye temperature $\Theta_{D}=374 \mathrm{~K}$ has been used for the calculation of the string potential. This is because we have found that the Molière potential gives the better result at $\theta_{\gamma}=0$ (i.e., the UFA limit) than the thermally averaged DoyleTurner potential. It should be emphasized that there is no free parameter in our numerical calculations. If we chose the potential so as to fit the UFA limit $\left(\theta_{\gamma}=0\right)$ with the experimental data, the agreements would have become even better.

In Fig. 5 we compare our theory with others. The dotted lines show the numerical results of the modified UFA expression given by Bă̌er, Katkov, and Strakhovenko (BKS), which has the lowest order correction term proportional to $\theta_{\gamma}^{2}$ [20]. As is expected, the BKS expression is applicable only in the region $\theta_{\gamma} \lesssim \theta_{0}$, where

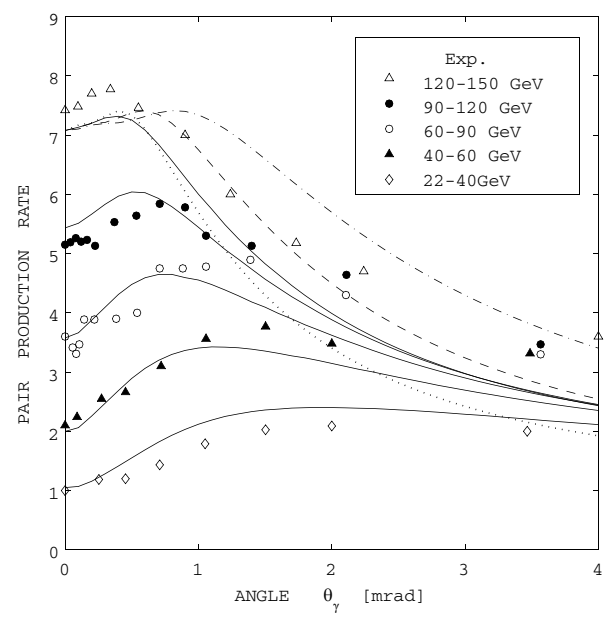

FIG. 4. Numerical results of crystal-assisted pair production in $\mathrm{Ge}\langle 110\rangle$. The symbols represent the experimental data [14]. No fitting parameter is included in the full calculations (solid lines). The dotted, dashed, and dashed-dottted lines represent the results based on the impact approximation. 


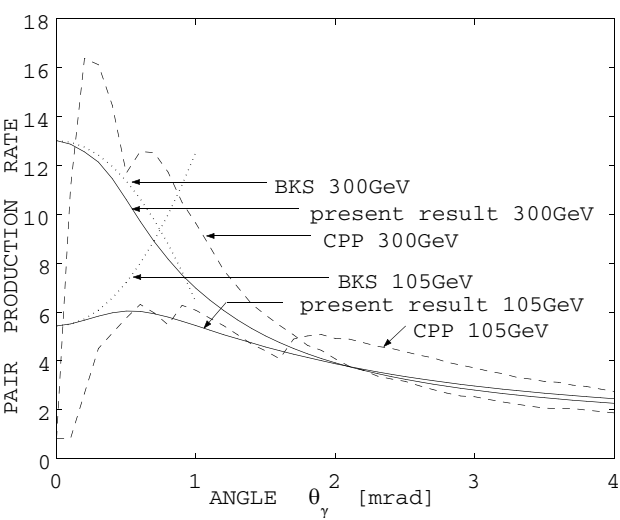

FIG. 5. Comparison with other theories. The dotted lines show the modified UFA theory [20] and the dashed lines show the CPP.

$\theta_{0}=U_{0} /\left(m_{0} c^{2}\right)$ represents the applicability of the Born approximation [20], $U_{0}$ being the height of the atomicstring potential. On the other hand, the numerical values of coherent pair production (CPP, the dashed lines) [23] follow the experimental data up to $\theta_{\gamma} \gtrsim \theta_{0}$. The peaks observed in Fig. 4 disappear at higher photon energies $\gtrsim$ $200 \mathrm{GeV}$, such as the line of $300 \mathrm{GeV}$. This is in agreement with the prediction in Ref. [20].

For the purpose of making an estimation as easy as possible, it is desirable to develop yet a simpler method of computation. The use of the "impact approximation" for calculating the parameter $\nu$ is suitable for such a purpose. In the impact approximation, the momentum change during collision is assumed to be proportional to the force multiplied by the interaction time $T$ so that we have $\mathbf{p}_{+\infty}-\mathbf{p}_{+\infty} \approx \mathbf{F}\left(\rho_{m}\right) T$, where $\rho_{m}$ is the impact parameter at the point of pair production, which is taken as the minimum distance to the string for a classical trajectory. The interaction time may be estimated as $T \sim$ $a\left(2 \rho_{m}\right) / v_{\perp}$, where $a$ is a free parameter of order 1 . Using $\gamma_{+} m c \boldsymbol{\beta}_{\perp \pm \infty}=\mathbf{p}_{ \pm \infty}$, we may calculate $\nu$ as

$$
\nu=\gamma_{+}\left|\frac{\boldsymbol{\beta}_{\perp+\infty}-\boldsymbol{\beta}_{\perp-\infty}}{2}\right|=a\left(\frac{F\left(\rho_{m}\right) \rho_{m}}{m c^{2} \theta_{\gamma}}\right) .
$$

Since Eq. (11) is determined only by the field at a certain point $\rho_{m}$ and the photon incident angle $\theta_{\gamma}$, we are ready to calculate Eq. (9).

The impact approximation is also convenient to see physics. The maximum value of field nonuniformity parameter $\nu_{\max }$ at a given $\theta_{\gamma}$ satisfies $\nu_{\max } \sim \theta_{0} / \theta_{\gamma}$ because $F\left(\rho_{m}\right) \rho_{m} \lesssim U_{0}$. Therefore, at $\theta_{\gamma}>\theta_{0}$ all the contribution comes from small $\nu$, while at $\theta_{\gamma}<\theta_{0}$ large $\nu$ contributes more and more as $\theta_{\gamma}$ decreases. It should be noted, however, that the contribution from $\nu<1$ is not negligible at $\theta_{L} \lesssim \theta_{\gamma}<\theta_{0}$.

In Fig. 4 we have shown the results of the impact approximation. The dotted line, dashed line, and dashed-dotted line represent $a=\sqrt{2} / 2, \sqrt{2}$, and $2 \sqrt{2}$, respectively. Calculations for other energies have shown similar behavior, suggesting that $a \approx \sqrt{2}$ gives reasonable agreements. Taking into account the simplicity of the impact approximation, the agreement seems enough for estimations in planning experiments as well as for evaluating pair-production rate in strong electromagnetic fields appearing in astrophysical problems. Thus, we may summarize that, if the motion of the produced pair is classical, the pair production by photons in the strong nonuniform field can be fairly well described by the simple standard expression, depending only on two Lorentz invariant parameters, $\chi$ and $\nu$.

[1] V. B. Berestetskii, E. M. Lifshitz and L. P. Pitaevskii, Quantum Electrodynamics (Pergamon, Oxford, 1982).

[2] Throughout this paper, "strong-field" means that it satisfies $\chi \gtrsim 1$, but we assume that the field is not too strong, so that the vacuum breakdown due to pair production is negligible.

[3] T. Erber, Rev. Mod. Phys. 38, 626 (1966).

[4] A. K. Harding, Radiat. Eff. Defects Solids 123, 625 (1991).

[5] C. D. Roberts, S. M. Schmidt, and D. V. Vinnik, Phys. Rev. Lett. 89, 153901 (2002), and references therein.

[6] C. Müller, A. B. Voitkiv, and N. Grün, Phys. Rev. Lett. 91, 223601 (2003).

[7] A. A. Sokolov and I. M. Ternov, Radiation from Relativistic Electrons, (AIP, New York, 1986).

[8] V. N. Bă̌er, and V. M. Katkov, Sov. Phys. JETP, 26, 854 (1968).

[9] V. N. Bă̌er, V. M. Katkov, and V. M. Strakhovenko, Electromagnetic Processes at High Energies in Oriented Single Crystals (World Scientific, Singapore, 1998).

[10] J. C. Kimball and N. Cue, Phys. Rep. 125, 69 (1985).

[11] K. Kirsebom et al., Phys. Rev. Lett. 87, 054801 (2001).

[12] J. Lindhard, K. Dan. Vidensk. Selsk. Mat. Fys. Medd. 34, No. 14 (1965).

[13] A. Belkacem, N. Cue, and J.C. Kimball, Phys. Lett. 111A, 86 (1985).

[14] A. Belkacem et al., Phys. Rev. Lett. 58, 1196 (1987).

[15] Yu. V. Kononets and I. S. Tupitsyn, JETP Lett. 57, 151 (1993).

[16] K. Kirsebom et al., Nucl. Instrum. Methods Phys. Res. Sect. B 135, 143 (1998).

[17] M. Kh. Khokonov and H. Nitta, Phys. Rev. Lett. 89, 094801 (2002).

[18] J. C. Kimball and N. Cue, Radiat. Eff. Defects Solids 122, 481 (1991).

[19] One may calculate the trajectory of an electron instead.

[20] V. N. Baĭer, V. M. Katkov, and V. M. Strakhovenko Sov. Phys. Usp. 32, 972 (1989).

[21] M. Kh. Khokonov, Phys. Scr. 55, 513 (1997).

[22] A. H. Sørensen, Nucl. Instrum. Methods Phys. Res. Sect. B 119, 1 (1996).

[23] G. Diambrini-Palazzi, Rev. Mod. Phys. 40, 611 (1968). 